\title{
Fetal gallstones: Antenatal detection and management
}

\author{
Deepti Naik \\ Dept. Of Radiodiagnosis and Imaging, M.S. Ramaiah Medical College and Hospitals, Bangalore, India \\ Email address: \\ drdeepti2004@hotmail.com \\ To cite this article: \\ Deepti Naik. Fetal Gallstones: Antenatal Detection and Management. International Journal of Medical Imaging. \\ Vol. 2, No. 6, 2014, pp. 146-148. doi: 10.11648/j.ijmi.20140206.15
}

\begin{abstract}
Fetal gallstones are a rare sonographic finding. We came across two cases of fetal gallstones detected in routine third trimester scan over a period of one year. Both cases were confirmed to have gallstones in postnatal scan and showed complete resolution of the gallstones at 35 and 45 days after birth. The presence of gallstones in the fetus does not alter the fetal prognosis or obstetrical management since complete resolution is seen in most of the cases in late third trimester or neonatal period which may be due to either spontaneous passage of gallstones during early neonatal period or dilution of cholesterol crystals with postnatal hydration.
\end{abstract}

Keywords: Antenatal, Gallstone, Ultrasound

\section{Introduction}

Fetal gallstones are an uncommon sonographic finding. Despite the increasing number of ultrasound scans performed during pregnancy, the prevalence as well as the risk factors are not well known. Till date no Indian data about fetal gall stones has been published in literature hence we decided to present the two cases of fetal gallstones which was diagnosed in our hospital over a period of one year. Two Radiologists with fifteen years and ten years of experience in antenatal ultrasonography had scanned both the cases mentioned below in order to confirm the presence of fetal gallstone.

\section{Case History}

\subsection{Case 1}

A 28 year old G1P0 patient was referred to our hospital for routine antenatal ultrasound scan at 30 weeks of gestation. An echogenic focus with distal acoustic shadowing was seen in the gall bladder of the fetus. No echogenic focus was seen in the fetal liver. No other fetal abnormality was seen. The fetal growth was normal and corresponding to the gestational age. There was no evidence of placental haemorrhage. A repeat antenatal ultrasound was performed at 32 weeks which showed the same findings of solitary fetal gallstone. The patient had a normal vaginal delivery at 38 weeks. The birth weight of the baby was $3100 \mathrm{~g}$. An abdominal ultrasound scan of the neonate was performed at 24 hours which confirmed the presence of solitary gall bladder stone. A follow up abdominal ultrasound scan of the child was done at 35 days which showed complete resolution of the gallstone. The child is now eight months old and is normal.

\subsection{Case 2}

A 30 year old G2P1 patient was referred to our hospital for antenatal scan at 34 weeks since hepatic calcifications in the fetus had been reported at another diagnostic centre. Multiple small echogenic foci with no distal acoustic shadowing was seen in the fetal gall bladder. No hepatic calcifications were identified. No other abnormality was seen in the fetus. The patient underwent normal vaginal delivery at 39 weeks of gestation. The birth weight of the baby was $3400 \mathrm{gms}$. Abdominal ultrasound scan of the neonate was done after 22 hours which confirmed the presence of multiple gallbladder stones. There was no dilatation of intrahepatic or extrahepatic biliary radicals. A follow up abdominal ultrasound scan was performed at 45 days which showed complete resolution of the fetal gallstones. The child is now four months old and is developing normally.

\subsubsection{Clinical History}

Both the pregnant women had no history of bleeding in the first or second trimester. No maternal abnormality was seen. There was no evidence of maternal gallstones. Postnatally, both the infants were clinically asymptomatic with no 
evidence of jaundice, pale colored stools or elevated serum bilirubin level. There was no evidence of hemolytic anemia or $\mathrm{Rh}(\mathrm{D})$ incompatibility in both the infants.

Images

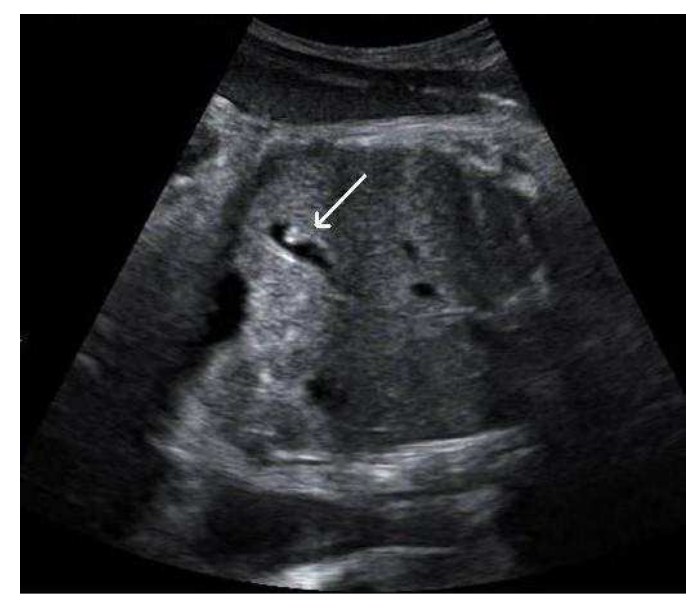

Figure 1 A. Antenatal ultrasound scan showing solitary gall bladder stone

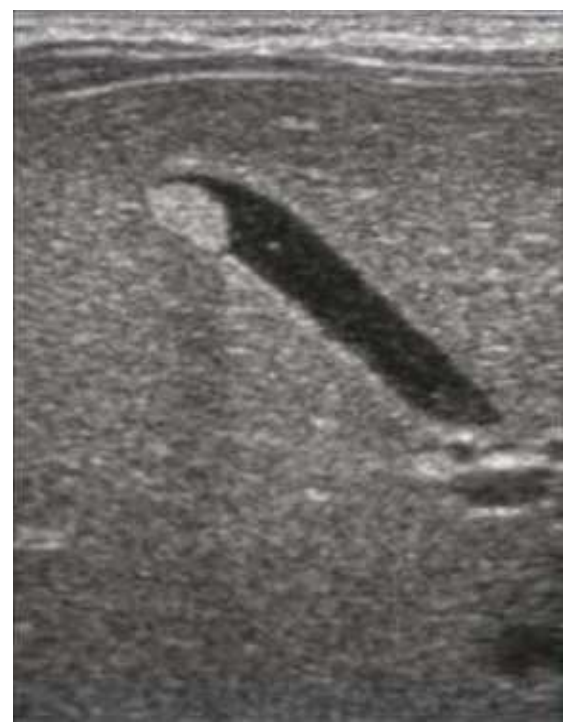

Figure 1 B. Postnatal ultrasound scan showing solitary gall bladder stone

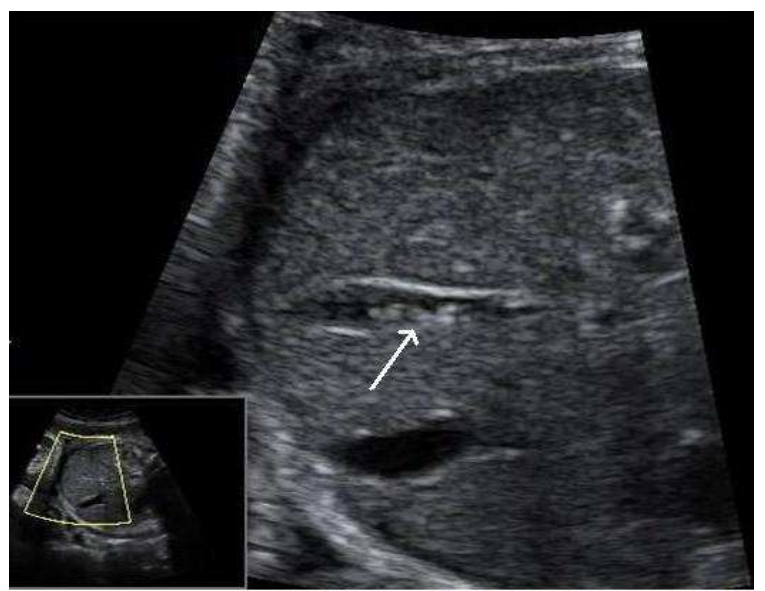

Figure 1 C. Antenatal ultrasound scan showing multiple gall bladder stones.

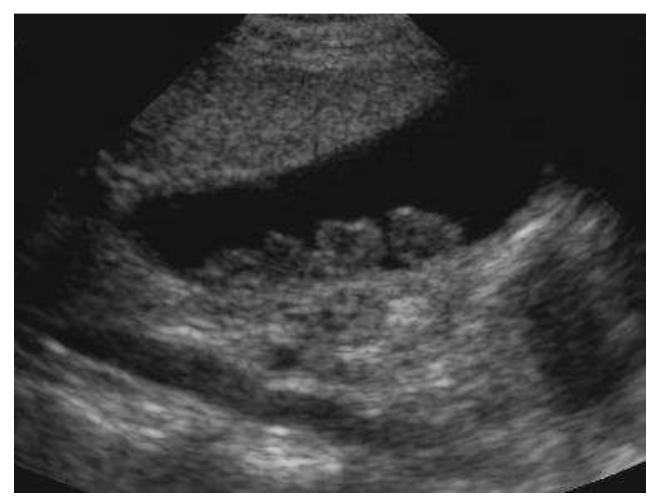

Figure 1 D. Postnatal ultrasound scan showing multiple gall bladder stones.

\section{Discussion}

Fetal gallstones are a rare sonographic finding in routine antenatal scans. Brown et al. ${ }^{[1]}$ have reported the largest series of fetal gallstones in 26 patients. Some studies have shown it to be more common in male fetus ${ }^{[2]}$. Complete resolution of fetal gallstones was observed in most of the cases in literature $^{[1,3]}$.

Fetal gallstones can be caused by haemolytic anemia, cholestasis, and maternal drug abuse ${ }^{[4]}$. Although these predisposing risk factors have been proposed to cause fetal gallstones, none have been conclusively proved ${ }^{[5]}$ Two possible explanations for formation of fetal gallstones have been proposed by Beretsky I and Lankin DH - (a) Presence of placental hematoma with subsequent breakdown of hemogolobin to bilirubin; or (b) Increased cholesterol secretion and depressed bile acid synthesis caused by estrogen. ${ }^{[6]}$ Fetal gallstones are usually observed in third trimester. ${ }^{[7]}$

Since the prognosis of isolated fetal gallstones is excellent ${ }^{[8]}$, familiarity with the range of appearance of gallstones in the fetus is important to avoid confusing them with potentially more serious pathological conditions like liver calcifications, calcified liver masses and meconium peritonitis.

Fetal gall bladder can be visualized in most of the routine antenatal ultrasound scans by angling the transducer caudally towards abdominal wall to the level of umbilical cord insertion which shows the gallbladder to the right of the umbilical vein. ${ }^{[8]}$ Fetal gallstones appear as single or multiple echogenic foci with or without acoustic shadowing. ${ }^{[1,8]}$ Distal shadowing is seen if calcium is present in the calculi. Non shadowing stones are predominantly composed of cholesterol crystals which may cause comet tail artifact ${ }^{[1]}$.

Calcifications in the fetal liver are a relatively more common finding. The site, size and distribution of the lesions are major factors in determining further management. Punctate echogenic lesions on the liver surface usually represent peritonial calcifications most commonly seen in meconium peritonitis. Isolated sub-capsular liver calcifications can be due to emboli from portal or hepatic veins. Liver calcifications can be single or multiple, which can be seen in TORCH infections. Calcified liver masses can 
be malignant lesions like hepatoblastoma or metastatic neuroblastoma $^{[4]}$.

In Meconium peritonitis scattered calcification are seen throughout the peritoneum. The calcification may be seen to line the liver but sometimes only a focal calcification with shadowing can also be seen. ${ }^{[4]}$

The risk factors proposed for fetal gallstones are hemolytic anemia, Rh (D) incompatibility, choledochal cysts, cholestasis, abruptio placenta and narcotic use during pregnancy. Two theories have been proposed for formation of fetal gallstones: (a) hemoglobin from placental hematoma transformed to bilirubin passes through placenta, increasing fetal indirect bilirubin level leading to formation of gallstones; (b) Increase in serum estrogen levels could increase the risk of pigmented gallstone formation by increasing the cholesterol excretion and decreasing biliary pigment synthesis. ${ }^{[1,8]}$

Many gallstones resolve spontaneously in-utero or soon after birth. Two hypotheses have been proposed for the disappearance of fetal gallstones - (a) spontaneous passage of gallstones during early neonatal period; or (b) dilution of cholesterol crystals with postnatal hydration ${ }^{[1]}$.

The presence of fetal gallstones does not alter fetal prognosis or obstetrical management since it gets resolved in late third trimester or neonatal period in most of the cases and is totally asymptomatic. ${ }^{[8]}$

Fetal gallstones are very rarely encountered in routine antenatal scan; but one should be familiar with the range of appearance of fetal gallstones in order to avoid confusing them with potentially more serious pathological conditions like liver calcification, calcified liver masses and meconium peritonitis.

\section{Conclusion}

Fetal gallstones are very rarely encountered in routine antenatal scan; but one should be familiar with the range of appearance of fetal gallstones in order to avoid confusing them with potentially more serious pathological conditions like liver calcification, calcified liver masses and meconium peritonitis.

\section{Acknowledgements}

D. Naik likes to thank Dr. M.G. Srinath and Dr. A. Ashok Kumar for their contribution to the case discussions and encouragement for this article.

\section{References}

[1] Brown DL, Teele RL, Doubilet PM, et al. Echogenic material in the fetal gallbladder: sonographic and clinical observations. Radiology 1992; 182:73-78

[2] Stringer-MD; Lim-P; Cave-M; et al. Fetal gallstones. JPediatr-Surg. 1996 Nov; 31(11): 1589-91.

[3] Klingensmith WC, Cioffi-Ragan DT. Fetal gallstones. Radiology 1988; 167:143-144

[4] McNamra Ann, Levine Deborah. Intrabdominal Fetal Echogenic Masses: A Practical guide to Diagnosis and Management. Radiographics 2005; 25:633-645

[5] Triunfo S, Rosati P, Ferrara P, Gatto A, Scambia G. Fetal Cholelithiasis: A Diagnostic Update and a Literature Review. Clinical Medicine Insights: Case Reports. 2013; 6:153-158

[6] Beretsky I, Lankin DH. Diagnosis of fetal cholelithiasis using real-time high resolution imaging employing digital detection. J Ultrasound med 1983; 2:381-383

[7] Bianchi DW, Crombleholme TM, D'Alton ME. Fetology, diagnosis \& management of the fetal patient. McGraw-Hill Professional. 2000 ISBN:0838525709

[8] Lariviere Michael. Fetal Cholelithiasis. J of Diagnostic Medical Sonography 2006; 22:403-406 